\title{
Editorial
}

\section{Computational Methods for High Energy Physics}

\author{
Carlo Cattani, ${ }^{1}$ Mehmet Bektasoglu, ${ }^{2}$ and Gongnan Xie ${ }^{3}$ \\ ${ }^{1}$ Department of Mathematics, University of Salerno, 84084 Fisciano (SA), Italy \\ ${ }^{2}$ Sakarya University, 54187 Sakarya, Turkey \\ ${ }^{3}$ Northwestern Polytechnical University, Xian 710072, China \\ Correspondence should be addressed to Carlo Cattani; ccattani@unisa.it
}

Received 31 March 2014; Accepted 31 March 2014; Published 24 April 2014

Copyright (C) 2014 Carlo Cattani et al. This is an open access article distributed under the Creative Commons Attribution License, which permits unrestricted use, distribution, and reproduction in any medium, provided the original work is properly cited. The publication of this article was funded by SCOAP $^{3}$.

New discoveries in high energy physics are leading to some intriguing and complex methods of analysis which demand for extremely advanced computational approaches. In order to face the complexity of the contemporary frontiers of Physics, computational methods and algorithms are becoming more and more important for a truthful interpretation of physical results. So that the computational tools are the key point for the proper understanding of physical results. Modern algorithms to be used in robust computer simulations and complex computations are then the pillars of the physical theory. Since the birth of the quantum theory, general relativity or astrophysical problems, researchers were dealing with problems where the physical evidence has been shown only as a consequence of advanced computational methods. Thus the computational approach became not only the most representative of any modern physical theory but also the epistemological setting of any modern physical theory.

Recent discoveries in high energy physics have opened new frontiers in Science and technologies, thus increasing the interests for new challenging computational methods. It should be noted that this interest is not restricted to high energy physics although many areas of modern physics is covered by this field. Research fields like cosmic rays, dark matter, neutrino physics, and so forth are subjects of interest for many scientists over the world. Together with experimental physics, computational methods like MonteCarlo simulations represent very important and useful tools for a better understanding of many phenomena. During the last decades, an increasing huge number of algorithms and simulation codes have been developed but not yet fully explored, from the point of view of their physicalmathematical consequences. Some of them are focusing on some general aspects of fundamental research (such as GEANT 4, FLUKA, etc.) while some others are mainly devoted to different areas of interest like CORSIKA, AIRES in cosmic rays theory and GENIE in neutrino physics. Each simulation code is based on several interaction models at high or low energy (e.g., QGSJET, EPOS, QGSP, etc.). Each model is based on the results obtained at different laboratories over the world, and the parameters are extrapolated to energies higher than an experiment could reach.

The main goal of this special issue is to bring together important information about modern simulation codes and to collect the main streams in the computational methods devoted to high energy problems, such as Monte-Carlo tools and interaction models. In this issue we have collected some original research papers on the frontier of computational physics covering a wide range of topics.

We have invited scientists active in the field to contribute with original research papers or review papers (both experimental and theoretical), which have included: Methods, algorithms, and codes, which have been discussed in the papers of F. Pop on "High performance numerical computing for high energy physics: a new challenge for big data science"; S. V. Chekanov and M. Erickson, on "A nonparametric peak finder algorithm and its application in searches for new physics"; M. Dima, "Computational determination of the dirac-theory adjunctator"; Y. Zhao, D. Baleanu, C. Cattani, De-Fu Cheng, and Xiao-Jun Yang, "Maxwell's equations on cantor sets: a local fractional approach"; Ming Li, S. C. Lim, C. Cattani, and M. Scalia, "Characteristic roots of a class of 
fractional oscillators"; Jinsong Leng, QixunGuo, and Tingzhu Huang, "The duals of fusion frames for experimental data transmission coding of high energy physics".

The paper of Turgay Korkut "FLUKA Monte Carlo simulations about cosmic rays interactions with kaidun meteorite", deals with Astrophysics and cosmic rays, while Cosmology is the main concerns of the papers of Alina Khaybullina, Ramil Izmailov, Kamal K. Nandi, and Carlo Cattani "Regular solutions in vacuum brans-dicke theory compared to vacuum Einstein theory", and Ming Li and Wei Zhao "Essay on Kolmogorov law of minus 5 over 3 viewed with golden ratio".

Related nonlinear mathematical models and methods and high energy questions, such as neutrino, photon, quantized energy, are discussed in the papers of Jun-Hui Kang, Ya-Zhou Wang, and Bao-Chun Li "Angular dependence of Meson production for different photon beam energies"; Mihai O. Dima, Yuri N. Pepelyshev, and Lachin Tayibov "Neutron noise analysis with flash-fourier algorithm at the IBR-2M reactor"; Bing Chen, Ke-Wei Wei, and Ailin Zhang " $\mathrm{X}(1870)$ and $\eta_{2}(1870)$ : which can be assigned as a hybrid state?"; Fu-Hu Liu, Ya-Hui Chen, Hua-Rong Wei, and BaoChun $\mathrm{Li}$ "Transverse momentum distributions of final-state particles produced in soft excitation process in high energy collisions"; Malika Betrouche, Mustapha Maamache, and Jeong Ryeol Choi, "Three-dimensional dirac oscillator with minimal length: novel phenomena for quantized energy"; Andreea Rodica Sterian, "Numerical simulations on nonlinear dynamics in lasers as related high energy physics phenomena"; Halil Arslan and Mehmet Bektasoglu, "Geant4 simulation study of deep underground muons: vertical intensity and angular distribution"; Bogdan Mitrica "Design study of an underground detector for measurements of the differential muon flux"; Cristian Toma, "Wavelets-computational aspects of sterian realistic approach to uncertainty principle in high energy physics: a transient approach"; Xian Wang, Hiroyuki Hirano, Gongnan Xie, and Ding Xu "VOF modeling and analysis of the segmented flow in Y-shaped microchannels for microreactor systems"; Alina-Mihaela Badescu and Alexandra Saftoiu, "Radio-wave propagation in salt domes: implications for a UHE cosmic neutrino detector".

Although a wide range of topics are touched upon, it is not possible to cover the entire field of computational methods in high energy physics into a single journal issue, also because of the continuous further progress both in the computational methods and in the high energy physics fields. We hope that this topics could be continued to track the updated trends years by years.

Finally, we would like to express our thanks to all the contributors of this special issue for their support and cooperation and to the qualified reviewers who helped the authors to improve the quality of their papers.

Carlo Cattani Mehmet Bektasoglu Gongnan Xie 

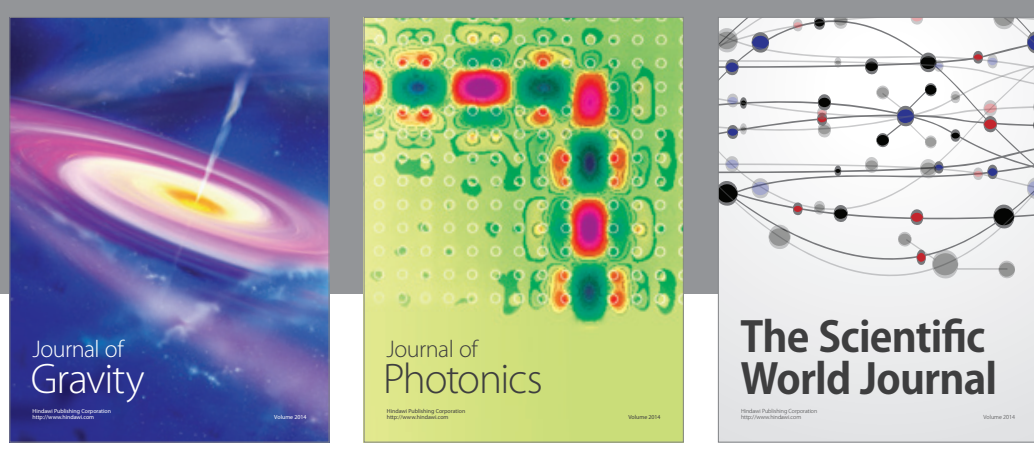

The Scientific World Journal
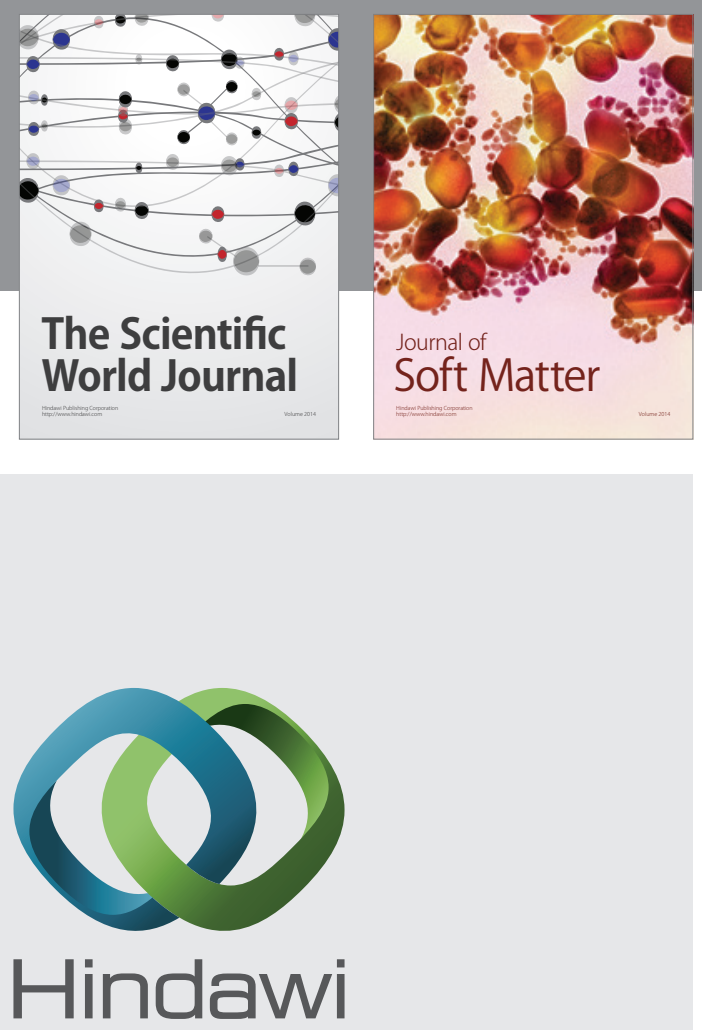

Submit your manuscripts at

http://www.hindawi.com

nternational Journal of

Statistical Mechanics
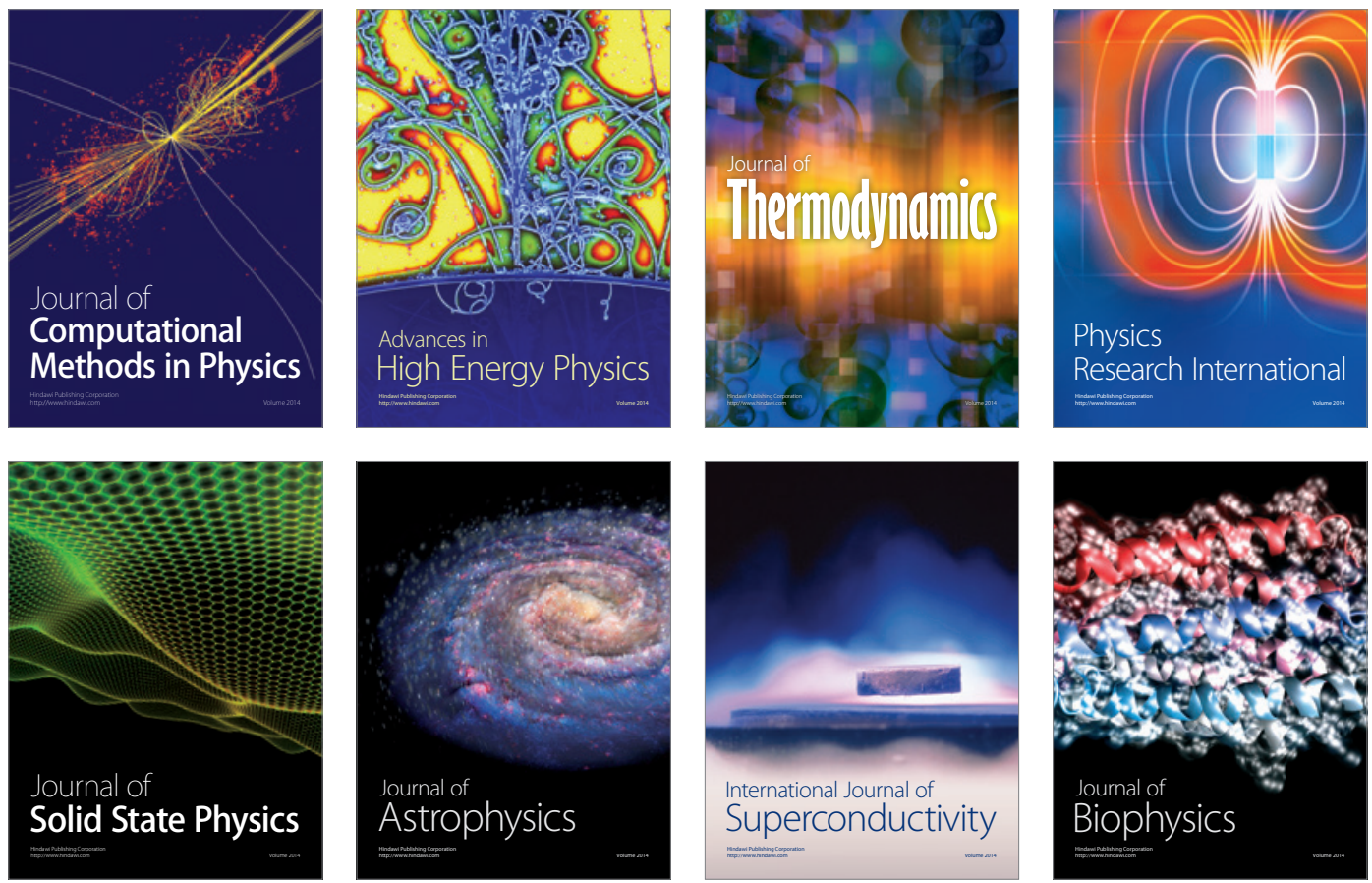
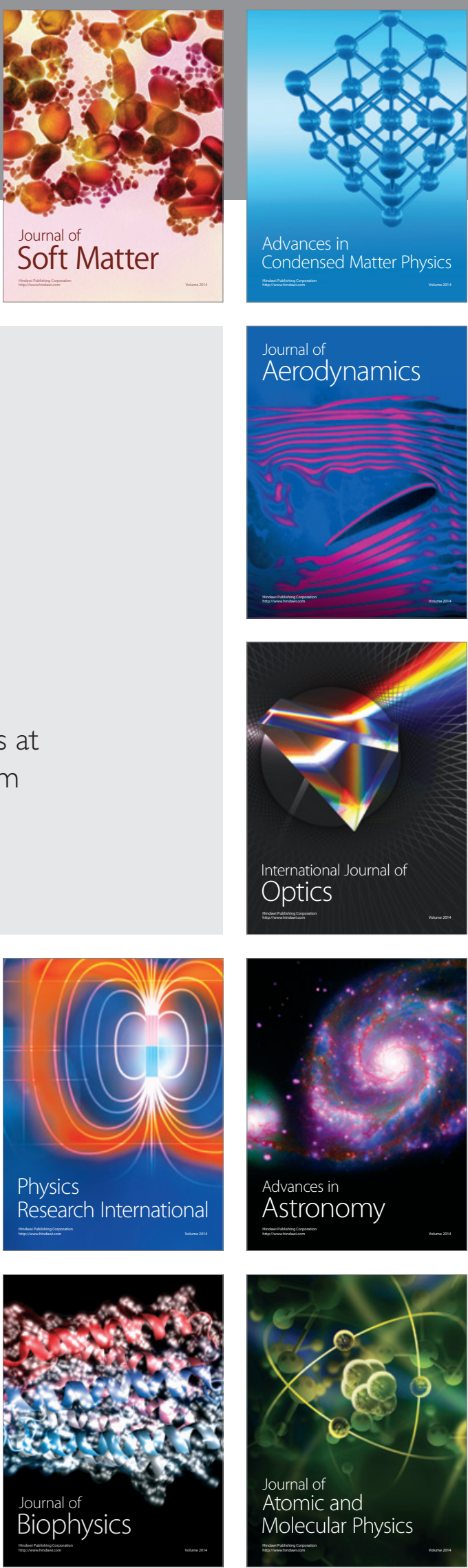